\title{
Dementia Care and the Role of Guideline Adherence in Primary Care: Cross-Sectional Findings From the DemTab Study
}

\section{Sonia Lech ( $\square$ sonia.lech@charite.de)}

Charité - Universitätsmedizin Berlin, corporate member of Freie Universität Berlin, Humboldt-Universität zu Berlin, Institute of Medical Sociology and Rehabilitation Science

\section{Julie Lorraine O'Sullivan}

Charité - Universitätsmedizin Berlin, corporate member of Freie Universität Berlin, Humboldt-Universität zu Berlin, Institute of Medical Sociology and Rehabilitation Science

\section{Johanna Drewelies}

Humboldt-Universität Berlin, Department of Psychology

\section{Wolfram Herrmann}

Charité - Universitätsmedizin Berlin, corporate member of Freie Universität Berlin, Humboldt-Universität zu Berlin, Institute of General Practice

\section{Robert Spang}

Technical University of Berlin

Jan-Niklas Voigt-Antons

Technical University of Berlin

Johanna Nordheim

Charité - Universitätsmedizin Berlin, corporate member of Freie Universität Berlin, Humboldt-Universität zu Berlin, Institute of Medical Sociology and Rehabilitation Science

\section{Paul Gellert}

Charité - Universitätsmedizin Berlin, corporate member of Freie Universität Berlin, Humboldt-Universität zu Berlin, Institute of Medical Sociology and Rehabilitation Science

\section{Research Article}

Keywords: dementia, primary care, adherence to dementia guideline

Posted Date: June 4th, 2021

DOl: https://doi.org/10.21203/rs.3.rs-546079/v1

License: (c) (i) This work is licensed under a Creative Commons Attribution 4.0 International License. Read Full License 
Version of Record: A version of this preprint was published at BMC Geriatrics on December 1st, 2021. See the published version at https://doi.org/10.1186/s12877-021-02650-8. 


\section{Abstract}

Background: General practitioners (GPs) play a key role in the care of people with dementia (PwD). However, the role of the German Dementia Guideline in primary care remains unclear. The main objective of the present study was to examine the role of guideline-based dementia care in general practices.

Methods: A cross-sectional analysis of data obtained from the DemTab study was conducted. Descriptive analyses of sociodemographic and clinical characteristics for GPs $(N=28)$ and PwD $(N=91)$ were conducted. Adherence to the German Dementia Guideline of GPs was measured at the level of PwD. Linear Mixed Models were used to analyze the associations between adherence to the German Dementia Guideline and GP factors at individual (age, years of experience as a GP, frequency of utilization of guideline, perceived usefulness of guideline) and structural (type of practice, total number of patients seen by a participating GP, and total number of PwD seen by a participating GP) levels as well as between adherence to the German Dementia Guideline and PwD's quality of life.

Results: Self-reported overall adherence of GPs was on average 71\% ( $S D=19.4$, range: $25-100)$. Adherence to specific recommendations varied widely (from $19.2 \%$ to $95.3 \%$ ) and the majority of GPs $(79.1 \%)$ reported the guideline as only partially or somewhat helpful. Further, we found lower adherence to be significantly associated with higher numbers of patients $(\gamma 10=-5.58, C l=-10.97,-0.19, p=.04)$. No association between adherence to the guideline and PwD's quality of life was found ( $\gamma 10=-.86, C l=-4.18$, $2.47, p=.61)$.

Conclusion: The present study examined the role of adherence to the German Dementia Guideline recommendations in primary care. Overall, GPs reported high levels of adherence. However, major differences across guideline recommendations were found. Findings highlight the importance of guidelines for the provision of care. Dementia guidelines for GPs need to be better tailored and addressed. Further, structural changes such as more time for PwD may contribute to a sustainable change of dementia care in primary care.

Trial registration: The DemTab trial was prospectively registered with the ISRCTN registry (Trial registration number: ISRCTN15854413). Registered 01 April 2019, https://doi.org/10.1186/ISRCTN15854413

\section{Background}

The current and imminent public health impact of dementia is vast. According to the World Alzheimer's Report published in 2015, 46.8 million people worldwide were estimated to live with dementia. Further, this number is estimated to increase to 74.7 million by 2030 and 131.5 million by $2050(1,2)$. Currently, about 1.7 million people with dementia (PwD) live in Germany, with a prevalence of $10 \%$ among older adults over the age of $65(3,4)$. Dementia not only affects those living with dementia, but also their families and informal caregivers, the health care system, and society as a whole (5-7). Consequently, policy makers and researchers are being urged to address dementia as a public health priority. In light of 
this, the World Health Organization (WHO) has called for national dementia strategies (6). WHO's recommendations for national areas of action include, amongst others, the improvement of dementia care delivery. In Germany, general practitioners (GPs) play a pivotal role in the management and delivery of care for PwD (8-13). For example, almost $99 \%$ of PwD living at home consult their GP at least once a year (14). Despite empirical evidence reporting that GPs acknowledge dementia care as a relevant topic and show positive attitudes towards the care of PwD, GPs find many aspects of dementia care to be challenging (15). The vast majority of previous research has focused on examining and improving primary care for dementia at an individual level of GPs. For example, research has primarily centered on providing knowledge training and education in diagnostics and dementia management (16-23). However, structural factors such as time constraints per patient $(17,24)$, as well as lack of cross-sectional collaboration $(25)$ and lack of social services support $(17,18)$ were frequently reported to negatively impact primary care delivery for dementia. It remains unclear which GP related factors impact dementia care delivery most.

Overall, evidence-based guidelines represent one public health tool that fosters optimal care delivery (26). Following recommendations of evidence-based guidelines may contribute to an improvement of dementia care $(27,28)$ and patient health-related quality of life $(29)$. In Germany, the German Dementia Guideline (28) provides evidence-based recommendations for treatment, care, and support of dementia. For example, the GDG recommends an intake of anti-dementia drugs dependent on type of dementia and severity of cognitive impairment. For individuals diagnosed with Alzheimer's Disease and a mild to moderate cognitive impairment, the intake of Acetylcholinesterase inhibitors is recommended (28). While the implementation of and adherence to dementia guideline recommendations may improve dementia care, little is known about the knowledge and utilization of the GDG among GPs in Germany. However, the GDG was not specifically developed for general practice. In addition, the associations between adherence to dementia guidelines (AGDG) and GP and PWD related factors remain unclear. The present study aims to explore the role of using the GDG in recommendations in primary care. First, we aim at describing a newly developed checklist assessing adherence to the GDG. Second, we aim to examine the association between AGDG and GP factors at individual levels (age, years of experience as a GP, frequency of utilization of the GDG, and perceived usefulness of the GDG) as well as at structural levels (type of practice, total number of patients seen by a participating GP during last three months, and total number of PwD seen by a participating GP during last three months). Based on previous literature, we hypothesize that structural factors will have a greater impact on AGDG than individual factors (Hypothesis 1). Third the association between AGDG and PwD's quality of life of will be examined. Based on previous literature (29), we expect a positive association between AGDG and quality of life (Hypothesis 2).

\section{Methods}

\section{Participants and recruitment}


This paper uses baseline data obtained from the DemTab study, a cluster randomized controlled trial (CRCT) that investigated the effects of a tablet-based intervention on guideline adherence (primary outcome) and health related PwD and informal caregiver outcomes (secondary outcomes) in the primary care setting. The study design and methods for the DemTab study have been published in detail elsewhere (30). In summary, the target population of the DemTab study were GPs, PwD, and their informal caregivers from Berlin and the surrounding area. For the purpose of the present study, only baseline data from GPs and PwD were included. Eligible GPs were currently operating GPs who provided informed consent to participate in the study. Eligible PwD were community living patients with a dementia diagnosis (ICD-10 F00-F03, G30, G31.0 and G31.82), who were treated in outpatient care and provided a signed informed consent to participate in the study (if he/she is still authorized to sign) or otherwise through a person holding the power of attorney.

\section{Study sample}

Due to the cluster-randomized design, the study sample was determined in two steps. In the first step, GPs were recruited through a variety of sampling methods: 1) advertisements in general practice related publications and newsletters through different networks in and around Berlin; 2) via phone recruitment of GPs randomly drawn from a database of the Statutory Health Insurance Physicians in Berlin, and 3) face-

to-face recruitment of GPs in their general practices in Berlin. In a second step, successfully recruited GPs recruited potentially eligible PwD from their practice. Overall, 629 GPs and 194 PwD were contacted for recruitment, of which 32 GPs and 102 PwD agreed to participate and signed an informed consent. On average, each GP referred about $7 \mathrm{PWD}$ (range: $1-17 ; \mathrm{mdn}=6 ; \mathrm{IQR}=3.5$ ) and successfully recruited about 4 PwD (range: $1-11 ; \mathrm{mdn}=3 ; \mathrm{IQR}=3.5$ ). The final sample consisted of $N=28 \mathrm{GPs}$ and $N=91 \mathrm{PwD}$. A flowchart is presented in Figure 1. A thorough description of the recruitment process and responses rates can be found in Lech et al. (31).

\section{Data collection}

Baseline data were collected from July 2019 to July 2020. Data from GPs were obtained through a questionnaire sent via mail. Data collection from PwD was originally planned and in most cases obtained by trained study nurses in the patient's home. However, due to the coronavirus disease 2019 (COVID-19), data collection was aligned with new regulations and changed from face-to-face assessment to phone interviews ( $n=12 \mathrm{PWD}$ ). The first assessment via phone was conducted on $30^{\text {th }}$ March 2020 . With the exception of the Mini Mental State Examination (MMSE), data collection via phone was uncomplicated and feasible. A follow up analysis revealed no differences in variables of PwD between data collected via face to face and data collected via phone interviews. However, due to the adjusted baseline data collection, it was not feasible to obtain data on the MMSE in a total of $11 \%$ of $\operatorname{PwD}(n=11)$. Additional information on the health and care situation of each PwD was obtained from GPs via another questionnaire.

\section{Measures}


At baseline, variables of interest were collected using a self-report questionnaire. A detailed description of all variables and measures can be found elsewhere (30). We aimed to obtain all self-reported items directly from the PwD. However, if the PwD was no longer able to provide answers or the validity of answers was questionable, answers/information were verified or obtained from the informal caregiver.

\section{Measures of adherence to German Dementia Guideline}

Adherence to the German Dementia Guideline (AGDG) was primarily assessed with a 23-item checklist. The checklist was developed based on the German Dementia Guideline (28) and other empirical work focusing on the role of guideline-based primary care (32-34). AGDG was self-reported by each GP on patient's level (for each participating PwD). The checklist can be found in German (original) and English (simple translation) in Appendix 1. The original checklist was composed in a dichotomous format with "yes" and "no" as options, but also included the category "not applicable". However, when analyzing the data, it became evident that the category "not applicable" was selected inconsistently. Specifically, because we failed to define "not applicable" a priori, it was unclear how this category was used. Consequently, there were known inconsistencies. In order to analyze the impact of the category "not applicable" and reduce possible bias in the calculation of the final AGDG score, we conducted a set of analyses to compare different scorings (see Appendix 2; Table A1). Scoring method 1: "not applicable" was recoded into missing data. Scoring method 2: "not applicable" was recoded into "not guideline adherent" (= 0), Scoring method 3: items, where "not applicable" was plausible were recoded into "not guideline adherent" $(=0)$, all other "not applicable" were recoded into missing data. Scoring method 4: items, where "not applicable" was plausible were recorded into "guideline adherent" $(=1)$, all other "not applicable" were recoded into missing data. For each scoring method, means and final scores were calculated (see Appendix 2; Table A1). Comparisons of means and correlations across scoring methods did not reveal any significant differences (see Appendix 2; Table A2 and Table A3). Due to conceptual assumptions, scoring method 1 was chosen for the calculation of the final score and "not applicable" was recoded as missing data. It is recommended for future research, when applying the present or any checklist for the assessment of guideline adherence, to define and include the category "not applicable" when appropriate, as this category may represent a valuable contribution. The final AGDG score for each PwD was calculated as the proportion of guideline adherence and all items answered ([sum of items answered as guideline adherent/sum of all answered items] x 100). The overall AGDG score was calculated as the mean percentage of per-patient guideline adherence across all GPs. The internal consistency of our scale for this data was Cronbachs's $\alpha=.876$.

In addition, we assessed other indicators measuring adherence to the GDG in primary care. First, knowledge of the guideline ("Are you familiar with the dementia guideline?"; yes/no), utilization of the guideline ("Do you use the dementia guideline?", yes/no), frequency of utilization ("How often do you use the guideline?"; always/often/sometimes/seldom/never) and perceived usefulness of the guideline ("How useful do you find the guideline?"; very/partially/somewhat/not helpful at all) were assessed from GPs. Further, prescribed anti-dementia drug (drug name), type of dementia (ICD-10 code) and cognitive status (MMSE) were compared based on guideline recommendations and a variable was computed $(0=$ 
not guideline adherent, 1 = guideline adherent, 2 = off-label use) to assess guideline adherence with regard to drug prescriptions.

\section{Measures of GPS and PWD}

Next, demographic and practice information was also collected for GPs. This information included age

(years), gender (female/male/other), years of experience as a GP (years), type of practice (single/shared), total number of patients seen by a participating GP during last three months (NPAT) and totalnumber of $P w D$ seen by a participating GP during last three months (NPWD).

Finally, sociodemographic information of PwD were collected, including age (years), gender (female/male/other), education (years of education) and living situation (alone/with partner/with caregiver/in outpatient facility). Further, level of care was measured according to the compulsory longterm care insurance in Germany (ranging from $1=$ low level of care to $5=$ high level of care) (35). Information on diagnostic procedure ("Who diagnosed the patient?"; current GP/other GP/specialist/other facility), type of dementia (ICD-10 code) and prescribed medications were obtained via GPs. Dementia related assessments included the Mini-Mental State Examination (total score ranges from 0 to 30, higher scores indicating higher cognitive status) (36). Quality of Life was assessed using the Quality of Life in Alzheimer's Disease questionnaire (QOL-AD, total score ranges from 13 to 52 , higher scores indicating better quality of life) (37).

\section{Statistical analysis}

First, descriptive analyses (means, standard deviations and ranges for continuous variables, frequencies for nominal and ordinal variables) of sociodemographic and clinical characteristics for GPs and PwD as well as for AGDG were calculated. Second, to address Hypothesis 1 and Hypothesis 2, Linear Mixed Models $(\mathrm{LMM})$ for continuous outcomes (covariance type $=$ variance components, estimation = Maximum Likelihood) were applied to analyze the predictive values of independent variables (level 1) accounting for the nested structure (GPs, level 2). The ID of GPs was used as a clustering variable. In step 1 , an intercept-only model (no level-one or level-two predictor was included in the model) was estimated to examine the variance associated between GP units and AGDG (base model). In order to describe dependencies due to the cluster structure of the data, an intraclass correlation coefficient (ICC) representing the ratio of the between-GP variance to the total variance was calculated. In step 2, a twolevel random-intercept model, which allows for variation in intercepts across GPs was estimated, in order to account for the clustered structure of the data. In order to explore the association between individual and structural factors and AGDG (Hypothesis 1), the following predictors were included in this model: 1) individual factors: age, years of experience as a GP, frequency of utilization of guideline and perceived usefulness of guideline, and 2) structural factors: type of practice, NPAT and NPWD. In order to examine the association between PwD's quality of life and AGDG (Hypothesis 2), quality of life was included as a predictor variable in another model. All predictors were standardized. The likelihood ratio (LR) test was used to compare the difference between the two nested models. All statistical analyses were performed 
using IBM SPSS Statistics for Windows V.27.0 and RStudio (Version 1.4.1106). All tests of significance were based on a $p<.05$ level and confidence interval of $95 \%$.

\section{Results}

\section{Characteristics of GPs}

Characteristics of participating GPs can be found in Table 1. Overall, $61.0 \%$ of participating GPs were female and on average 50 years old ( $S D=7.99$, range: 38-67), with a mean of about 12 years of experience as a GP $(S D=9.11$, range: $1-29)$. Less than half of GPs $(n=12,42.9 \%)$ were working in a single-handed practice. On average, GPs treated $N=1489$ patients $(S D=656.03$, range: $700-2990)$ and $\mathrm{N}=61 \mathrm{PwD}(S D=52.80$, range: $9-200)$ during the last three months.

Table 1

Main characteristics of GPS

\begin{tabular}{|llllll|}
\hline Sociodemographic characteristics & N & $\%$ & $M$ & $S D$ & range \\
\hline Age & 28 & & 49.9 & 98.0 & $38-67$ \\
\hline Gender (female) & 17 & 60.7 & & & \\
\hline Years of experience as a GP & 28 & & 11.8 & 9.1 & $1-29$ \\
\hline Single-handed practice (yes) & 12 & 42.9 & & & \\
\hline NPAT & 28 & & 1488.9 & 656.0 & $700-2990$ \\
\hline NPWD & 28 & 60.9 & 52.8 & $9-200$ \\
\hline $\begin{array}{l}\text { Note. } N=28 \text { GPs, } M=\text { Mean, } S D=\text { Standard Deviation, NPAT = total number of patients seen by a } \\
\text { participating GP during last three months, NPWD = total number of PwD seen by a participating GP } \\
\text { during last three months. }\end{array}$ \\
\hline
\end{tabular}

\section{Characteristics of PwD}

Table 2 presents an overview of PwD's main characteristics. Overall, almost $60 \%(n=54)$ of PwD were female, were on average 80 years old $(S D=6.3$, range: $63-94)$, and reported an average of 12.6 years of education ( $S D=3.3$, range: 8 - 17). More than half of PwD lived together with their spouse or partner $(n=$ $53 ; 58.2 \%$ ). About $51 \%$ of PwD were in need of substantial care (care level 3 or higher). About half of PwD obtained their dementia diagnosis from a specialist (55.2\%) and about a third (33.3\%) from a GP. PwD visited their GP on average 2.8 times in the last three months $(S D=1.9$, range: $0-11)$. The mean MMSE score was $18.9(S D=7.8$, range: $0-30)$ and the majority of $\operatorname{PwD}(n=38 ; 51.4 \%)$ were mildly cognitive impaired. More than one third of PwD (36.7\%) reported the intake of an anti-dementia drug. The mean QOL-AD score was 34.1 ( $S D=5.8$, range: $18-48$ ). 
Table 2

Main characteristics of PwD

\begin{tabular}{|c|c|c|c|c|c|}
\hline Sociodemographic characteristics & $n$ & $\%$ & $M$ & $S D$ & range \\
\hline Age & & & 80.5 & 6.3 & $63-94$ \\
\hline Gender (female) & 54 & 59.3 & & & \\
\hline Years of education & & & 12.7 & 2.8 & $8-17$ \\
\hline \multicolumn{6}{|l|}{ Living situation } \\
\hline Alone & 17 & 18.7 & & & \\
\hline With spouse/partner & 53 & 58.2 & & & \\
\hline With another informal caregiver & 6 & 6.6 & & & \\
\hline In outpatient facility & 15 & 16.5 & & & \\
\hline Care level (yes) & 71 & 64.6 & & & \\
\hline Care level 1 & 5 & 5.5 & & & \\
\hline Care level 2 & 20 & 22.0 & & & \\
\hline Care level 3 & 29 & 31.9 & & & \\
\hline Care level 4 or 5 & 17 & 18.7 & & & \\
\hline \multicolumn{6}{|l|}{ Dementia related assessments } \\
\hline \multicolumn{6}{|l|}{ Diagnostic procedure } \\
\hline Current GP & 16 & 18.4 & & & \\
\hline Other GP & 13 & 14.9 & & & \\
\hline Ambulatory specialist (psychiatrist, neurologist) & 48 & 55.2 & & & \\
\hline Other facility & 10 & 11.4 & & & \\
\hline \multicolumn{6}{|l|}{ Type of dementia diagnosis } \\
\hline Alzheimer's Disease & 34 & 37.4 & & & \\
\hline Unspecified dementia & 32 & 35.2 & & & \\
\hline Vascular dementia & 17 & 18.7 & & & \\
\hline Other type of dementia diagnosis & 7 & 7.7 & & & \\
\hline MMSE score & & & 18.9 & 7.8 & $0-30$ \\
\hline
\end{tabular}

Note. $\mathrm{N}=91, \mathrm{M}=$ Mean, $\mathrm{SD}=$ Standard Deviation, $\mathrm{MMSE}=$ Mini Mental State Examination, $\mathrm{QOL}-\mathrm{AD}=$ Quality of Life in Alzheimer's Disease questionnaire. 
Severity of cognitive impairment

Mild

$38 \quad 51.4$

Moderate

$27 \quad 36.5$

Severe

$\begin{array}{ll}9 & 12.2\end{array}$

Intake of anti-dementia drugs (yes)

$33 \quad 36.7$

QOL-AD

34.1

5.8

$18-48$

Note. $\mathrm{N}=91, M=$ Mean, $\mathrm{SD}=$ Standard Deviation, $\mathrm{MMSE}=$ Mini Mental State Examination, $\mathrm{QOL}-\mathrm{AD}=$ Quality of Life in Alzheimer's Disease questionnaire.

\section{The role of the GDG in primary care}

The overall mean AGDG score was $71.02(S D=19.4$, range: $25-100)$. Table 3 shows overall frequencies of adherence (yes) for all recommendations. 
Table 3

Guideline adherence over all PwD on item level

\begin{tabular}{|c|c|c|}
\hline Checklist items (yes) & $n$ & $\%$ \\
\hline Basic geriatric assessment & 82 & 93.2 \\
\hline \multicolumn{3}{|l|}{ Diagnostics } \\
\hline Physical examination and psychopathological/psychiatric evaluation & 86 & 94.3 \\
\hline Laboratory tests & 83 & 94.3 \\
\hline Differential diagnostics & 64 & 76.2 \\
\hline Cognitive and neuropsychological tests & 66 & 78.6 \\
\hline Recent medical history & 68 & 74.7 \\
\hline CT/MRI scans & 59 & 72.8 \\
\hline GP administered cognitive screening & 46 & 54.1 \\
\hline Assessment of further physical impairments/medical conditions & 79 & 87.8 \\
\hline Assessment of further mental health impairments/psychiatric conditions & 66 & 74.2 \\
\hline Psychological and behavioral symptoms of dementia & 75 & 91.5 \\
\hline Assessment of entire current medication & 81 & 95.3 \\
\hline Pharmacological treatment options & 53 & 65.4 \\
\hline Discussion of non-pharmacological interventions & 61 & 72.6 \\
\hline Prescription of non-pharmacological interventions & 46 & 54.8 \\
\hline Involvement of dementia specialist & 52 & 61.9 \\
\hline Information about Care services & 54 & 64.3 \\
\hline Information about local support services & 41 & 54.7 \\
\hline Care plan developed & 45 & 54.9 \\
\hline Daily activities & 65 & 79.3 \\
\hline Self-help measures & 58 & 69.0 \\
\hline Risks assessed and discussed & 50 & 62.5 \\
\hline Driving skills & 29 & 49.2 \\
\hline Care level situation & 63 & 79.7 \\
\hline
\end{tabular}




\begin{tabular}{|lcc|}
\hline Rights and the availability of local advocacy services & 55 & 67.9 \\
\hline Palliative care options & 14 & 19.2 \\
\hline Caregiver stress level & 60 & 74.1 \\
\hline Caregiver resources and support offers & 53 & 87.1 \\
\hline Note. $N=28 G P s, N=91$ PwD. & & \\
\hline
\end{tabular}

The great majority of GPs reported following the guideline recommendations with regard to assessing a patient's entire current medication plan (95.3\%), physical and psychopathological evaluations (94.3\%), laboratory tests as part of the diagnostics procedure (94.3\%), conducting a basic geriatric assessment (93.2\%), and assessing psychological and behavioral symptoms of dementia (91.5\%). Recommendations on discussing palliative care $(19.2 \%)$ or the current driving situation $(49.2 \%)$, as well as obtaining CT/MRI scans as part of the diagnostic procedure (54.1\%), providing of information about local support services (54.8\%) and prescribing non-pharmacological interventions (54.8\%) were less frequently followed.

Further, the great majority $(n=20 ; 71.4 \%)$ of GPs reported to be familiar with the GDG, but only $19.2 \%$ ( $n=$ 5) reported using it often. Further, $20.8 \%(n=5)$ reported the GDG as very helpful and $45.8 \%(n=11)$ found it to be partially helpful. Almost one third $(n=8 ; 28.6 \%)$ reported the length of the GDG as a reason for not using the guideline. See Table 4 for a complete breakdown of attitudes toward the GDG. With regard to anti-dementia drug prescription, $10.3 \%$ of PwD were prescribed a drug that was not in line with guideline recommendations, and $44.8 \%$ were prescribed a drug that was considered as off-label use by the GDG. 
Table 4

GPs attitudes toward the German Dementia Guideline

\begin{tabular}{|lll|}
\hline Attitudes & $n$ & $\%$ \\
\hline Knowledge about guideline (yes) & 20 & 71.4 \\
\hline Utilization of guideline (yes) & 17 & 60.7 \\
\hline Frequency of utilization & 26 & \\
\hline Often & 5 & 19.2 \\
\hline Sometimes & 10 & 35.7 \\
\hline Seldom & 7 & 26.9 \\
\hline Never & 4 & 15.4 \\
\hline Helpfulness of guideline & 24 & \\
\hline Very & 5 & 20.8 \\
\hline Partially & 11 & 45.8 \\
\hline Somewhat & 8 & 33.3 \\
\hline Not helpful at all & 0 & 0 \\
\hline Reasons for non-utilization of guideline & 14 & \\
\hline Length & 8 & 28.6 \\
\hline Lack of relevance & 4 & 14.3 \\
\hline Lack of knowledge & 2 & 7.1 \\
\hline Note: $N=28$ GPs. & & \\
\hline
\end{tabular}

\section{Association between AGDG and factors on GP and PwD level}

Results of the intercept-only model (base model) indicated there was statistically significant variation in the intercepts (ICC $=.536$ ), accounting for approximately $54 \%$ of the variance in AGDG and indicating a substantial clustering of observations within level 2 units. With regard to Hypothesis 1, the regression coefficient for NPAT showed a negative and significant predictive relationship between NPAT and AGDG ( $\gamma 10=-5.58, C l=-10.97,-0.19, p=.04$ ), indicating an association between higher number of patients and lower AGDG scores. Age ( $\mathrm{\gamma} 10=-7.39, C l=-19.81,5.03, p=.23)$, Years of experience as a $G P(\gamma 10=7.92, C l$ $=-5.03,20.86, p=.22)$, frequency of utilization of $G D G(\gamma 10=-2.06, C l=-12.05,7.93, p=.68)$ and perceived usefulness of $G D G(\mathrm{Y} 10=2.78, C l=-5.71,11.29, p=.51)$ as well as type of practice $(\mathrm{Y} 10=-2.54$, 
$C l=-7.73,2.65, p=.33)$ and $N P W D(\gamma 10=-3.26, C l=-9.06,2.53, p=.26)$ were not significant in predicting AGDG. Further, results of the likelihood ratio test showed a significant increase of the fit by adding level 1 predictors $\left(\chi^{2}=155.6, d f=7, p<.001\right)$. With regard to Hypothesis 2 , the regression coefficient for QOL-AD shows no significant association between QOL-AD and AGDG score $(\gamma 10=-.86, C l=-4.18,2.47, p=.61)$.

\section{Discussion}

The main objective of the present study was to examine the role of the German Dementia Guideline in primary care. Main findings indicate overall high levels of AGDG, although large differences can be observed across recommendations. With regard to Hypothesis 1 , the total number of patients seen by a participating GP during the last three months was significantly and negatively associated with AGDG. With regard to Hypothesis 2, quality of life was not significantly associated with AGDG.

\section{Adherence to the German Dementia Guideline in primary care}

For the purpose of this study, a checklist was developed to examine the role of adherence to the German Dementia Guideline. This checklist facilitates the assessment of AGDG for research (calculation of AGDG score) and may assist GPs in daily practice with treatment and care of dementia. With regard to the AGDG score, present findings indicate a relatively high overall guideline adherence among participating GPs. In contrast to our study, a study examining the effect of a disease management intervention on guideline adherence reported a much lower overall mean score of guideline adherence (38). However, Vickrey et al. (2006) obtained information on guideline adherence by medical record review as well as by caregiver survey. The present study measured AGDG primarily with a self-report checklist. While the overall AGDG was relatively high, variability between recommendations were found. With regard to palliative care, past research has frequently acknowledged, that due to the progressive nature of dementia, advance care planning and palliative care is important, and GPs play a key role in the in-time planning (39-41). In order to ensure and respect preferences and wishes of PwD, it is recommended to ascertain their views in an early stage of the disease, before ability to consider the future is limited (42). As the majority of community dwelling PwD receive regular care from their GPs and GPs often have a longstanding relationship with their PwD, GPs are particularly suited to address palliative care(43). In order to improve advanced and palliative care planning, we recommend that dementia guidelines should include guidance and recommendations on that matter. With regard to the present result on fitness to driving, the GDG specifically provides a section on dementia and driving and outlines the importance of evaluating current driving skills with the progression of dementia (28). Previous research has acknowledged, that GPs play a key role in the assessment of fitness to drive in dementia, a topic of uncertainty and conflict for GPs (44). A recent study found that GPs discussed fitness to drive with only $32.1 \%$ of potentially driving elderly patients (45). Previous studies indicated that fitness to drive is severely impaired in moderate and severe dementia (46). In sum, there is an urgent need to develop and 
provide training and guidance on performance of driving assessments for GPs so that they are able to perform such assessments with PwD (47).

In addition to the AGDG score, we have analyzed data on anti-dementia drug intake with regard to guideline adherence. The GDG recommends intake of anti-dementia drugs dependent on type of dementia and severity of cognitive impairment. For example, for individuals diagnosed with Alzheimer's Disease and a mild to moderate cognitive impairment, the intake of Acetylcholinesterase inhibitors is recommended (28). In the present sample, about $37 \%$ of PwD reported the intake of an anti-dementia drug, a finding in line with previous studies $(48,49)$. For example, a study on medical treatment of PwD in Germany reported $25 \%$ of ambulatory PwD receiving an anti-dementia drug, and found that this number varied depending on whether PwD were seen by a GP and specialist or solely a GP ( $48 \%$ versus $24.5 \%$, respectively) (50). Past research has consistently reported a positive association between involvement of a GP/specialist and anti-dementia drug prescription $(49,51)$. In the present study, about $10 \%$ of PwD reported the intake of an anti-dementia drug which was not in line with GDG recommendations, and almost half (44.5\%) reported an intake of off-label drugs. With regard to medication, based on present findings, the prescription of anti-dementia drugs requires improvement. An anti-dementia drug treatment should be always based on individual assessments of risks and benefits $(28,48)$. Key dementia care providers, especially GPs, should have knowledge on the latest guideline recommendations regarding anti-dementia drugs and their risks and benefits. Collaborative care models may improve anti-dementia drug prescriptions in ambulatory care for PwD. Our data shows that the majority of PwD (62\%) saw a specialist in addition to their GP. Collaborations between GPs and specialists (e.g., psychiatrist or neurologist) can improve the implementation of guideline recommendations with regard to anti-dementia drugs $(48,52-55)$.

Finally, nearly one third of GPs who participated in this study reported length of the GDG as a reason for non-utilization of the guideline and another third of GPs reported the guideline as only somewhat helpful. Given these findings, research should reconsider the current format of the GDG for GPs. More compiled and practical guidelines are needed. Further, it is of great importance to include perspectives and recommendations from general practice in the guideline development. Although the GDG acknowledged the important role of GPs in the care of PwD (28), the German College of General Practitioners and Family Physicians was hardly involved in the development of the guideline. The validity of the current GDG expired in February 2021. Thus, a new guideline is currently being developed. We highly recommend including the perspectives and experiences of GPs in the development and implementation of the new GDG.

\section{Associations between AGDG and variables on GP and PwD level}

It was of great interest to examine the associations between AGDG and factors on GPs and PwD level. With regard to individual and structural factors of GPs and AGDG, results of multilevel analyses revealed that only the total number of patients seen by a participating GP during the last three months were 
negatively associated with AGDG. This finding is partially in line with previous empirical work. While it is widely believed that more time per patient improves patient's health and quality of care (56-58), a systematic review of clinical trials found insufficient empirical evidence that patients benefit from longer consultations (59). However, with regard to dementia, past research has recommended more time in primary care for PwD (12). In Germany, a recent study found an average consultation length of 7.6 minutes (60). It is reasonable to believe that GPs with larger patient loads have less time to spend with each patient, consequently resulting in less time to focus on and follow guideline recommendations. However, the observed effect should be interpreted with caution. The present study has no data on the frequency of visits for each patient nor the total number of hours GPs actually spend with their patients. Future research is needed in order to gain a better understanding of the role of a GPs patient load, time spent with each patient, and time spent on patient care, and its impact on guideline adherence.

With regard to the association between AGDG and PwD's quality of life, no significant association was found. This finding is not consistent with previous research that examined the effects of a dementia guideline-based disease management program in a CRCT and found significant improvements in healthrelated quality of life in PwDs $(29,61)$. Future research is required in order to gain a better understanding of the role of guideline-based dementia care in primary practices (62). The present paper is based on baseline data collected within a CRCT that aims to evaluate the effect of a technology-based intervention on AGDG in primary care. We are currently conducting follow-up assessments with GPs and PwD and will be able to conduct a more in-depth examination of the association between AGDG and GP and PWD in the near future.

\section{Limitations}

This is the first study in Germany assessing adherence to the German Dementia Guideline in primary care with a checklist developed based on the GDG recommendations. However, there is a number of limitations that must be outlined. First, the present sample is drawn from a cRCT examining a tabletbased intervention for GPs, PwD, and their informal caregivers. The DemTab study is, inter alia, based on a convenience sample. Specifically, participants self-selected to participate in the study, which may propose a higher risk of biased data. Participants' decision to participate may be correlated with traits that affect the study (63). For example, the high guideline adherence found in our study may be because participating GPs were particularly engaged and interested in the study's topic. Second, with regard to the assessment of guideline adherence, the post hoc recoding of the category "not applicable" as missing data must be addressed. Even if comparisons of means and correlations across scoring methods did not reveal any significant differences, it is possible that data labeled as "not applicable" can provide further insight into responses and, therefore, may represent a valuable contribution in the assessment of AGDG. Future research, when using the present or any checklist to assess guideline adherence, should define and include this category. Third, with regard to the checklist, guideline adherence in the present study was measured with a self-report checklist. A potential rater bias (systematic introduction of variance by GPs) has to be kept in mind when interpreting the scores of guideline adherence in the present study $(64,65)$. Future research should assess guideline adherence in a more objective manner. Last, with regard to 
Hypothesis 1 and Hypothesis 2, due to the cross-sectional design we cannot draw causal relationships. Future studies should examine longitudinal data in order to examine multidirectional associations between guideline adherence and GP and PwD related variables.

\section{Conclusion}

The present study examined the role of adherence to the German Dementia Guideline provided by GPs in Germany. Overall adherence to the guideline was relatively high. However, major differences between recommendations were observed. In order to ensure and improve primary care of PwD, dementia guidelines for GPs need to be better tailored and addressed. Further, collaborative care between GPs and specialists as well as structural changes, such as GPs spending more patient time with PwD, may contribute to a sustainable change of dementia care in primary care.

\section{List Of Abbreviations}

AGDG adherence to dementia guideline

CRCT cluster randomized trial

GDG German Dementia Guideline

GP general practitioner

ICC interclass correlation coefficient

Mdn Median

MMSE Mini Mental State Examination

NPAT total number of patients seen by a participating GP during last three months

NPWD total number of PwD seen by a participating GP during last three months

PwD people with dementia

SD standard deviation

UK United Kingdom

QOL-AD Quality of Life in Alzheimer's Disease questionnaire

\section{Declarations}

\section{Ethics approval and consent to participate}


Ethical approval was obtained by the ethics committee of the Charité - Universitätsmedizin Berlin (EA1/085/19). Written informed consent was obtained from all participants or legal guardians prior to data collection.

\section{Consent for publication}

Not applicable

\section{Availability of data and materials}

Data is stored in a non-publicly available repository. Data are however available from the corresponding author on request.

\section{Competing interests}

The authors declare that they have no competing interests.

\section{Funding}

The research is funded by the Innovation Fund of the Federal Joint Committee (G-BA), (Grant Project “DemTab” Grant-Number 01VSF17039).

\section{Authors' contributions}

SL was primary contributor to data analysis, data interpretation and manuscript drafting. JLOS, JD, WH, JNVA, RS assisted with the writing and interpretation of findings. PG supervised the analysis. JN, PG and JNVA designed and led the DemTab study in which the present study was embedded. All authors contributed to the present study and critically revised and approved the final manuscript.

\section{Acknowledgements}

The authors extend their special appreciation to Sebastian Möller and Adelheid Kuhlmey who designed the DemTab study. Further, we would like to thank all our participating GP practices in Berlin and surrounding area. Finally, we would like to thank Alesia Woszidlo for proofreading this manuscript.

\section{References}

1. Livingston G, Sommerlad A, Orgeta V, Costafreda SG, Huntley J, Ames D, et al. Dementia prevention, intervention, and care. The Lancet. 2017;390(10113):2673-734.

2. Prince MJ. World Alzheimer Report 2015: the global impact of dementia: an analysis of prevalence, incidence, cost and trends: Alzheimer's Disease International; 2015.

3. Deutsche Alzheimer Gesellschaft. Die Haufigkeit von Demenzerkrankungen.; 2019.

4. Thyrian JR, Stentzel U. Estimating the Number of People with Dementia in Germany in 2030 on County Level. Psychiatr Prax. 2020. 
5. Winblad B, Amouyel P, Andrieu S, Ballard C, Brayne C, Brodaty H, et al. Defeating Alzheimer's disease and other dementias: a priority for European science and society. The Lancet Neurology. 2016;15(5):455-532.

6. World Health Organization. Dementia: a public health priority. World Health Organization. World Health Organization; 2012.

7. Zeisel J, Bennett K, Fleming R. World Alzheimer Report 2020: Design, dignity, dementia: Dementiarelated design and the built environment. 2020.

8. Leve V, Ilse K, Ufert M, Wilm S, Pentzek M. Driving and dementia : An issue for general practice?! Zeitschrift fur Gerontologie und Geriatrie. 2017;50(Suppl 2):55-62.

9. Pentzek M, Vollmar HC, Wilm S, Leve V. Putting dementia awareness into general practice. Zeitschrift fur Gerontologie und Geriatrie. 2017;50(2):44-7.

10. Pentzek M, Fuchs A, Abholz H-H, Wollny A. Awareness of local dementia services among general practitioners with academic affiliation. Aging Clinical and Experimental Research. 2011;23(3):241-3.

11. Pentzek M, Abholz HH, Ostapczuk M, Altiner A, Wollny A, Fuchs A. Dementia knowledge among general practitioners: first results and psychometric properties of a new instrument. International psychogeriatrics. 2009;21(6):1105-15.

12. Thyrian JR, Hoffmann W. Dementia care and general physicians-a survey on prevalence, means, attitudes and recommendations. Central European journal of public health. 2012;20(4):270-5.

13. Kaduszkiewicz H, Wiese B, van den Bussche H. Self-reported competence, attitude and approach of physicians towards patients with dementia in ambulatory care: results of a postal survey. BMC health services research. 2008;8:54.

14. Schwarzkopf L, Menn P, Leidl R, Wunder S, Mehlig H, Marx P, et al. Excess costs of dementia disorders and the role of age and gender - an analysis of German health and long-term care insurance claims data. BMC health services research. 2012;12(1):165.

15. Wang M, Xu X, Huang Y, Shao S, Chen X, Li J, et al. Knowledge, attitudes and skills of dementia care in general practice: a cross-sectional study in primary health settings in Beijing, China. BMC Family Practice. 2020;21(1):89.

16. Foley T, Boyle S, Jennings A, Smithson WH. "We're certainly not in our comfort zone": a qualitative study of GPs' dementia-care educational needs. BMC Family Practice. 2017;18(1):66.

17. Turner S, lliffe S, Downs M, Wilcock J, Bryans M, Levin E, et al. General practitioners' knowledge, confidence and attitudes in the diagnosis and management of dementia. Age and Ageing. 2004;33(5):461-7.

18. Koch T, lliffe S. Rapid appraisal of barriers to the diagnosis and management of patients with dementia in primary care: a systematic review. BMC Family Practice. 2010;11:52.

19. Mitchell AJ, Meader N, Pentzek M. Clinical recognition of dementia and cognitive impairment in primary care: a meta-analysis of physician accuracy. Acta psychiatrica Scandinavica. 2011;124(3):165-83. 
20. Olafsdottir M, Foldevi M, Marcusson J. Dementia in primary care: why the low detection rate? Scandinavian journal of primary health care. 2001;19(3):194-8.

21. Tang EYH, Birdi R, Robinson L. Attitudes to diagnosis and management in dementia care: views of future general practitioners. International psychogeriatrics. 2018;30(3):425-30.

22. Stewart TV, Loskutova N, Galliher JM, Warshaw GA, Coombs LJ, Staton EW, et al. Practice Patterns, Beliefs, and Perceived Barriers to Care Regarding Dementia: A Report from the American Academy of Family Physicians (AAFP) National Research Network. The Journal of the American Board of Family Medicine. 2014;27(2):275-83.

23. Mukadam N, Cooper C, Kherani N, Livingston G. A systematic review of interventions to detect dementia or cognitive impairment. Int J Geriatr Psychiatry. 2015;30(1):32-45.

24. van den Dungen P, van Marwijk HW, van der Horst HE, Moll van Charante EP, Macneil Vroomen J, van de Ven PM, et al. The accuracy of family physicians' dementia diagnoses at different stages of dementia: a systematic review. Int J Geriatr Psychiatry. 2012;27(4):342-54.

25. Franz CE, Barker JC, Kim K, Flores Y, Jenkins C, Kravitz RL, et al. When Help Becomes a Hindrance: Mental Health Referral Systems as Barriers to Care for Primary Care Physicians Treating Patients With Alzheimer's Disease. American Association for Geriatric Psychiatry. 2010.

26. World Health Organization. Delivering quality health services: a global imperative for universal health coverage. 2018.

27. Jeschke E, Ostermann T, Vollmar HC, Tabali M, Schad F, Matthes H. Prescribing patterns in dementia: a multicentre observational study in a German network of CAM physicians. BMC Neurology. 2011;11(1):99.

28. Deutsche Gesellschaft für Psychiatrie und Psychotherapie, Psychosomatik und Nervenheilkunde, Deutsche Gesellschaft für Neurologie. S3 Leitlinie „Demenzen“ (Langversion - Januar 2016). 2016.

29. Vickrey B, Mittman B, Connor K, Pearson M, Della Penna R, Ganiats T, et al. The effect of a disease management intervention on quality and outcomes of dementia care: a randomized, controlled trial. Annals of internal medicine2006. p. 713-26.

30. Lech S, O'Sullivan JL, Gellert P, Voigt-Antons JN, Greinacher R, Nordheim J. Tablet-Based Outpatient Care for People With Dementia. GeroPsych. 2019;32(3):135-44.

31. Lech S, O'Sullivan JL, Wellmann L, Supplieth J, Döpfmer S, Gellert P, et al. Recruiting general practitioners and patients with dementia into a cluster randomised controlled trial: strategies, barriers and facilitators. BMC Med Res Methodol. 2021;21(1):61.

32. Downs M, Cook A, Rae C, Collins KE. Caring for patients with dementia: The GP perspective. Aging \& Mental Health. 2000;4(4):301-4.

33. Murphy K, O'Connor DA, Browning CJ, French SD, Michie S, Francis JJ, et al. Understanding diagnosis and management of dementia and guideline implementation in general practice: a qualitative study using the theoretical domains framework. Implementation Science. 2014;9(1):1-13.

34. Vickrey B, Mittman B, Connor K, Pearson M, Della Penna R, Ganiats T, et al. The effect of a disease management intervention on quality and outcomes of dementia care: a randomized, controlled trial. 
Annals of internal medicine. 2006;145(10):713-26.

35. Federal Ministry of Health. Peer review on “Germany's latest reforms of the 730 long-term care system”: Host Country Discussion Paper - Germany. DG 731 Employment, Social Affairs and Inclusion. European Commission. https://ec.europa.eu/social/main.jsp?

langld=en\&catld=89\&newsld=9008. Accessed 22 April 2021. 2018.

36. Folstein MF, Folstein SE, McHugh PR. “Mini-mental state”. A practical method for grading the cognitive state of patients for the clinician. J Psychiatr Res. 1975;12.

37. Logsdon RG, Gibbons LE, McCurry SM, Teri L. Assessing Quality of Life in Older Adults With Cognitive Impairment. Psychosomatic Medicine. 2002;64(3):510-9.

38. Vickrey B, Mittman B, Connor K, Pearson M, Della Penna R, Ganiats T, et al. The effect of a disease management intervention on quality and outcomes of dementia care: a randomized, controlled trial. Annals of internal medicine. 2006;145(10):713-26.

39. van der Steen JT, Radbruch L, Hertogh CMPM, de Boer ME, Hughes JC, Larkin P, et al. White paper defining optimal palliative care in older people with dementia: A Delphi study and recommendations from the European Association for Palliative Care. Palliative Medicine. 2013;28(3):197-209.

40. Evans N, Pasman HRW, Donker GA, Deliens L, Van den Block L, Onwuteaka-Philipsen B. End-of-life care in general practice: A cross-sectional, retrospective survey of 'cancer', 'organ failure' and 'oldage/dementia' patients. Palliative Medicine. 2014;28(7):965-75.

41. Tilburgs B, Vernooij-Dassen $M$, Koopmans R, van Gennip H, Engels $Y$, Perry $M$. Barriers and facilitators for GPs in dementia advance care planning: A systematic integrative review. PLoS One. 2018;13(6):e0198535.

42. Dening KH, Jones L, Sampson EL. Preferences for end-of-life care: a nominal group study of people with dementia and their family carers. Palliat Med. 2013;27(5):409-17.

43. Schers $H$, van den Hoogen $H$, Bor $H$, Grol R, van den Bosch W. Familiarity with a GP and patients' evaluations of care. A cross-sectional study. Fam Pract. 2005;22(1):15-9.

44. Pentzek M, Michel JV, Ufert M, Vollmar HC, Wilm S, Leve V. Fahrtauglichkeit bei DemenzTheoretische Rahmung und Konzept einer Vorgehensempfehlung für die Hausarztpraxis. Zeitschrift für Evidenz, Fortbildung und Qualität im Gesundheitswesen. 2015;109(2):115-23.

45. Leve V, Pentzek M, Fuchs A, Bickel H, Weeg D, Weyerer S, et al. GPs' awareness of car driving among oldest patients: exploratory results from a primary care cohort. BJGP open. 2021.

46. Toepper M, Falkenstein M. Driving fitness in different forms of dementia: an update. Journal of the American Geriatrics Society. 2019;67(10):2186-92.

47. Walsh L, Chacko E, Cheung G. The process of determining driving safety in people with dementia: $A$ review of the literature and guidelines from 5 English speaking countries. Australasian Psychiatry. 2019;27(5):480-5.

48. Wucherer D, Eichler T, Kilimann I, Hertel J, Michalowsky B, Thyrian JR, et al. Antidementia drug treatment in people screened positive for dementia in primary care. Journal of Alzheimer's Disease. 2015;44(3):1015-21.

Page $21 / 24$ 
49. Schütz LH, Boronat-Garrido X, Moser FA, Suhr R, Lahmann N. Dementia-specific drug treatment in home care settings: A German multicentre study. Journal of Clinical Nursing. 2019;28(5-6):862-9.

50. Bohlken J, Schulz M, Rapp MA, Bätzing-Feigenbaum J. Pharmacotherapy of dementia in Germany: Results from a nationwide claims database. European Neuropsychopharmacology. 2015;25(12):2333-8.

51. Hoffmann F, van den Bussche H, Wiese B, Schön G, Koller D, Eisele M, et al. Impact of geriatric comorbidity and polypharmacy on cholinesterase inhibitors prescribing in dementia. BMC psychiatry. 2011;11(1):1-8.

52. Schulz M, Bohlken J, Schulz M, Hering R, von Stillfried D, Bätzing-Freigenbaum J. Medikamentöse Behandlung von Patienten mit Demenz unter besonderer Berücksichti-gung regionaler Versorgungsunterschiede. Zentralinstitut für die kassenärztliche Versorgung in Deutschland. 2015.

53. Thyrian JR, Eichler T, Pooch A, Albuerne K, Dreier A, Michalowsky B, et al. Systematic, early identification of dementia and dementia care management are highly appreciated by general physicians in primary care-results within a cluster-randomized-controlled trial (DelpHi). Journal of multidisciplinary healthcare. 2016;9:183.

54. Schulz M, Bohlken J, Hering R, Bätzing-Feigenbaum J. Diagnostische und therapeutische Leistungsdichte von neu erkrankten, zu Hause lebenden Patienten mit Demenz. VersorgungsatlasBericht Available online: http://wwwversorgungsatlasde/themen/alle-analysen-nach-datum-sortiert. 2014.

55. Laver K, Cations M, Radisic G, de la Perrelle L, Woodman R, Fitzgerald JA, et al. Improving adherence to guideline recommendations in dementia care through establishing a quality improvement collaborative of agents of change: an interrupted time series study. Implementation Science Communications. 2020;1(1):80.

56. Mercer SW, Fitzpatrick B, Guthrie B, Fenwick E, Grieve E, Lawson K, et al. The CARE Plus study - a whole-system intervention to improve quality of life of primary care patients with multimorbidity in areas of high socioeconomic deprivation: exploratory cluster randomised controlled trial and costutility analysis. BMC Med. 2016;14(1):88.

57. Hutton C, Gunn J. Do longer consultations improve the management of psychological problems in general practice? A systematic literature review. BMC health services research. 2007;7:71-.

58. Mercer SW, Fitzpatrick B, Gourlay G, Vojt G, McConnachie A, Watt GCM. More time for complex consultations in a high-deprivation practice is associated with increased patient enablement. $\mathrm{Br} \mathrm{J}$ Gen Pract. 2007;57(545):960-6.

59. Wilson AD, Childs S. Effects of interventions aimed at changing the length of primary care physicians' consultation. Cochrane Database Syst Rev. 2006(1):Cd003540.

60. Irving G, Neves AL, Dambha-Miller H, Oishi A, Tagashira H, Verho A, et al. International variations in primary care physician consultation time: a systematic review of 67 countries. BMJ Open. 2017;7(10):e017902. 
61. Pond D, Mate K, Stocks N, Gunn J, Disler P, Magin P, et al. Effectiveness of a peer-mediated educational intervention in improving general practitioner diagnostic assessment and management of dementia: a cluster randomised controlled trial. BMJ Open. 2018;8(8):e021125.

62. Joling KJ, van Eenoo L, Vetrano DL, Smaardijk VR, Declercq A, Onder G, et al. Quality indicators for community care for older people: A systematic review. PLoS One. 2018;13(1):e0190298.

63. Ellenberg JH. Selection bias in observational and experimental studies. Stat Med. 1994;13(5-7):55767.

64. Hoyt WT. Rater bias in psychological research: When is it a problem and what can we do about it? Psychological methods. 2000;5(1):64.

65. Blumenthal-Barby JS, Krieger $\mathrm{H}$. Cognitive biases and heuristics in medical decision making: a critical review using a systematic search strategy. Medical Decision Making. 2015;35(4):539-57.

\section{Figures}

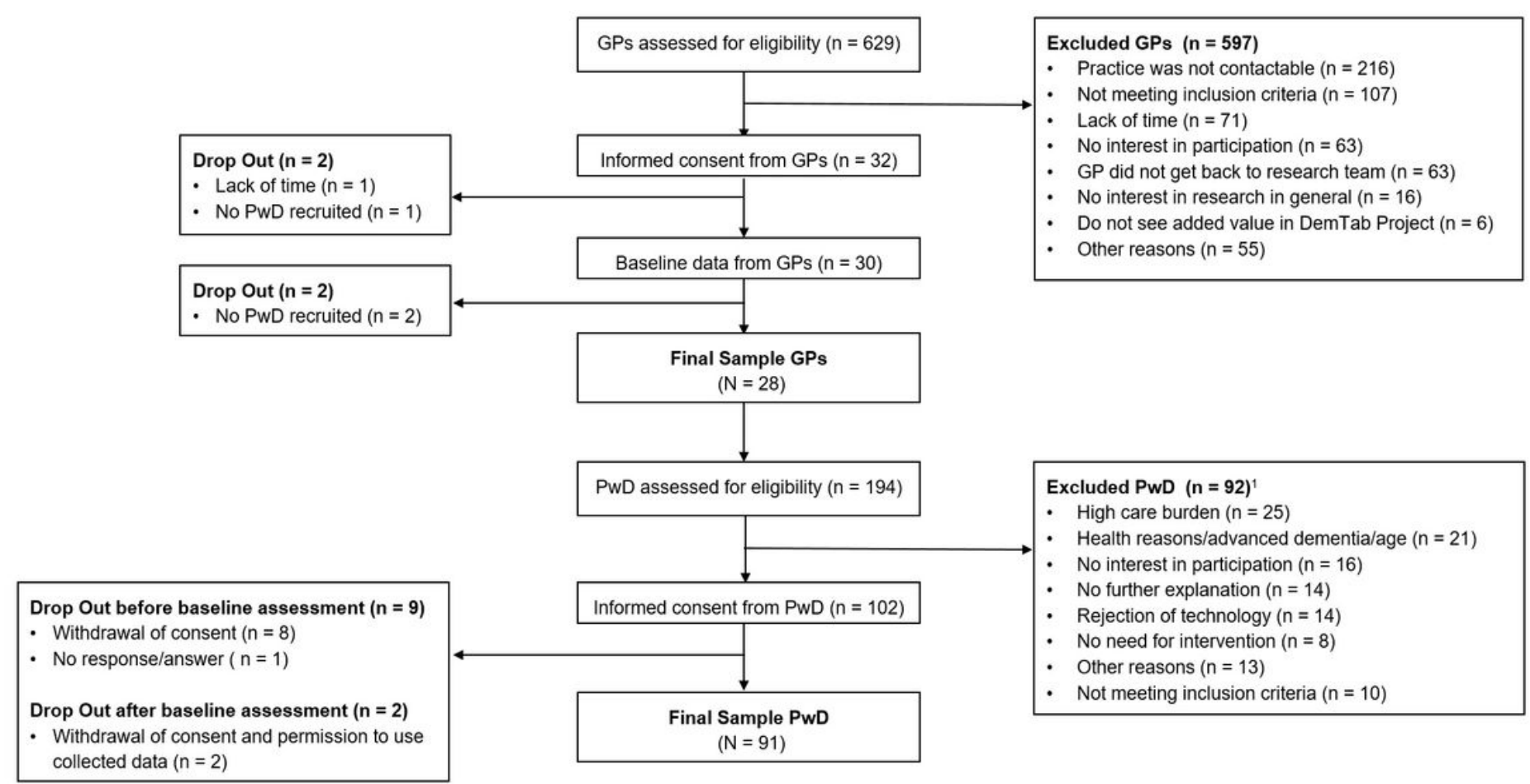

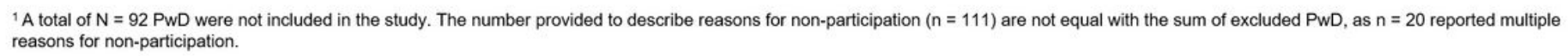

Figure 1

Flow chart of recruitment

\section{Supplementary Files}

This is a list of supplementary files associated with this preprint. Click to download. 
- Additionalfile1.pdf

- Additionalfile2.pdf

Page 24/24 